\title{
Randomised controlled study of oral erythromycin for treatment of gastrointestinal dysmotility in preterm infants
} P C Ng, K W So, K S C Fung, C H Lee, T F Fok, E Wong, W Wong, K L Cheung,
A F B Cheng

\begin{abstract}
Aim-To evaluate the effectiveness of oral erythromycin as a prokinetic agent for the treatment of moderately severe gastrointestinal dysmotility in preterm very low birthweight infants.

Methods-A prospective, double blind, randomised, placebo controlled study in a tertiary referral centre of a university teaching hospital was conducted on 56 preterm infants $(<1500 \mathrm{~g})$ consecutively admitted to the neonatal unit. The infants were randomly allocated by minimisation to receive oral erythromycin $(12.5 \mathrm{mg} / \mathrm{kg}$, every six hours for 14 days) or an equivalent volume of placebo solution (normal saline) if they received less than half the total daily fluid intake or less than 75 $\mathrm{ml} / \mathrm{kg} /$ day of milk feeds by the enteral route on day 14 of life. The times taken to establish half, three quarters, and full enteral feeding after the drug treatment were compared between the two groups. Potential adverse effects of oral erythromycin and complications associated with parenteral nutrition were assessed as secondary outcomes.
\end{abstract}

Results-Twenty seven and 29 infants received oral erythromycin and placebo solution respectively. The times taken to establish half, three quarters, and full enteral feeding after the drug treatment were significantly shorter in the group receiving oral erythromycin than in those receiving the placebo $(p<0.05, p<0.05$ and $p<0.0001$ respectively). There was also a trend suggesting that more infants with prolonged feed intolerance developed cholestatic jaundice in the placebo than in the oral erythromycin group (10 $v 5$ infants). None of the infants receiving oral erythromycin developed cardiac dysrhythmia, pyloric stenosis, or septicaemia caused by multiresistant organisms.

Conclusions-Oral erythromycin is effective in facilitating enteral feeding in preterm very low birthweight infants with moderately severe gastrointestinal dysmotility. Treated infants can achieve full enteral feeding 10 days earlier, and this may result in a substantial saving on hyperalimentation. However, until the safety of erythromycin has been confirmed in preterm infants, this treatment modality should remain experimental.
Prophylactic or routine use of this medication for treatment of mild cases of gastrointestinal dysmotility is probably not warranted at this stage.

(Arch Dis Child Fetal Neonatal Ed 2001;84:F177-F182)

Keywords: erythromycin; gastrointestinal dysmotility; enteral feeding; preterm; very low birthweight infants; randomised controlled trial

Erythromycin, a commonly used macrolide antibiotic, has been found to possess potent prokinetic properties and therefore enhances gastrointestinal motor activity. ${ }^{1-3}$ Its motilin agonist action has been clinically exploited for the treatment of patients with chronic functional pseudo-obstruction, ${ }^{4}$ gastro-oesophageal reflux ${ }^{5}$ postoperative intestinal dysmotility, ${ }^{6}$ gastroparesis secondary to diabetes, ${ }^{7}$ and scleroderma, ${ }^{8}$ and after surgical vagotomy. ${ }^{9}$ Erythromycin can also promote antroduodenal coordination and has been used in facilitating the transpyloric passage of endoscope,${ }^{10}$ Watson's capsule, ${ }^{11}$ and nasoenteric feeding devices into the proximal small bowel. ${ }^{12}$ Recently, its use as a prokinetic agent has further been extended to preterm very low birthweight (VLBW) infants for the management of non-anatomically obstructive gastrointestinal dysmotility. We and others have reported successful treatment of severe cases with intravenous and oral preparations of the drug. ${ }^{13-16} \mathrm{~A}$ review of the statistics of our neonatal unit during the oral vancomycin trial indicated that infants under $1500 \mathrm{~g}$ often experienced protracted feed intolerance, and the median ages of achieving full enteral feeding were 27 and 28 days in the oral vancomycin and placebo group respectively. ${ }^{17}$ As prolonged hyperalimentation has been associated with increased risks of serious and sometimes even life threatening complications, including cholestatic jaundice and liver impairment, nutritional deficiency, biochemical rickets, catheter related septicaemia, and pain, as well as anaesthetic risks from repeated intravenous and long line insertion, ${ }^{13}$ prokinetic therapy can be considered for improving the gastrointestinal motility in selected preterm infants who have failed to establish full enteral feeding after an extended period and in whom an anatomically obstructive lesion of the gastrointestinal tract has been excluded. This prospective, randomised, placebo controlled study aims to evaluate the effectiveness of oral erythromycin as a prokinetic agent in promoting enteral feeding in preterm VLBW infants who have moderately severe gastrointestinal dysmotility. 
The potential adverse effects of erythromycin and complications from hyperalimentation are assessed as secondary outcomes.

\section{Patients and methods}

PATIENTS

Preterm infants consecutively admitted to the neonatal unit at Prince of Wales Hospital, Hong Kong between November, 1998 and May 2000 were eligible for inclusion in the study if they satisfied the following criteria: birth weight less than $1500 \mathrm{~g}$; received less than half the total daily fluid intake or less than 75 $\mathrm{ml} / \mathrm{kg} /$ day of milk feeds by the enteral route on day 14 of postnatal age; parental consent to be entered into the study. The following infants were excluded: those with lethal congenital anomalies; those with anatomical gastrointestinal abnormalities, such as oesophageal atresia, intestinal stenosis or atresia, Hirschsprung's disease or necrotising enterocolitis; those with congenital cyanotic heart diseases; and those who had received major gastrointestinal surgery within the first two weeks of life.

SAMPLE SIZE

Our unit statistics revealed a consistently high prevalence of milk intolerance during the three consecutive years before the study. Over $75 \%$ of VLBW infants did not receive full enteral milk feeding by day 14 of life. The mean (SD) age at which full enteral feeding was achieved in the latter group of patients was 35 (18.4) days. In addition, our previous experience in using oral erythromycin suggested that most infants with uncomplicated gastrointestinal dysmotility could achieve full enteral feeding by between 5 and 10 days after initiation of the drug treatment. It was determined that 28 infants would be required in each arm of a randomised controlled study to detect a significant difference at the $5 \%$ level with a power of $80 \%$, if the mean age of achieving full enteral feeding was to decrease from 35 days to 21 days. As about $65-70$ VLBW infants were admitted to the neonatal unit each year and the predicted rate of consent from the parents approached $80 \%$, we expected to complete the study within 18 months.

\section{RANDOMISATION}

Eligible infants were randomly assigned by computer on day 14 to receive either oral erythromycin or normal saline. A designated member of staff not involved in the clinical care of the newborns performed the randomisation by minimising three important variables: gestational age; birth weight; age of introduction of milk feeds. The attending neonatal team caring for the infants was unaware of the randomisation assignments.

DRUG

The oral route of drug administration was preferred to the intravenous route because all life threatening and fatal cardiac complications related to erythromycin had been associated with the parenteral route of drug delivery. ${ }^{18} 19$ Typically, the peak serum drug concentration achieved after an intravenous infusion of erythromycin is $4-10$ times higher than when the drug is administered by the oral route. ${ }^{20-22}$ As the safety dose limit of intravenous erythromycin in preterm infants has not yet been determined, ${ }^{23}$ administration by this route is best avoided. With regard to the dose of oral erythromycin, we opted to use a slightly higher dose $(12.5 \mathrm{mg} / \mathrm{kg} / \mathrm{dose})$ than the lower dose regimen (3-5 mg/kg/dose) ${ }^{1415}$ commonly used for the management of gastrointestinal dysmotility because: (a) our previous success in treatment of severe gastrointestinal dysmotility in preterm infants indicated that the higher dosage was effective ${ }^{13} ;(b)$ former studies using a lower dose regimen often required large intravenous loading doses of erythromycin (15-30 mg/kg/ day) for the initial few days of treatment ${ }^{14}{ }^{15} ;(c)$ the serum drug concentration achieved by oral medication is likely to be lower than that achieved by the intravenous route ${ }^{20-22} ;(d)$ larger doses of erythromycin have also been shown to facilitate gastric emptying by stimulating postprandial antroduodenal motor activity. ${ }^{24}$ Infants allocated to receive active drug were given oral erythromycin (Ery-Ped; erythromycin ethyl succinate diluted to $12.5 \mathrm{mg} / \mathrm{ml}$ with sterile water; Abbott Laboratories, Abbott Park, Illinois, USA) $12.5 \mathrm{mg} / \mathrm{kg}$, every six hours. Those allocated to receive placebo solution were given an equivalent volume of normal saline. As the oral erythromycin preparation is a white liquid suspension, both active drug and normal saline were mixed thoroughly into the milk feeds to mask their appearance from the attending clinical team. This procedure was performed by two designated staff not involved in the clinical management of these infants at that time. In addition, the oral drug was identified only by a code number to ensure effective blinding. The drug treatment was started on day 15 of postnatal age immediately after randomisation, and all patients received a 14 day course of treatment. When oral feeding had to be discontinued after the start of the study, all oral mediations including erythromycin and placebo solution were also suspended. Administration of the study solutions was resumed after the infant was restarted on enteral nutrition. During the study period, the use of other prokinetic agents such as cisapride, levosulpiride, and metoclopramide was strictly prohibited. Electrocardiography was performed immediately before and on the second week of drug treatment for the measurement of QT intervals.

ENTERAL AND PARENTERAL NUTRITION All VLBW infants were started on parenteral nutrition (6\% TrophAmine (McGaw Inc, Irvine, California, USA) and 20\% Intralipid (Kabi Pharmacia AB, Stockholm, Sweden)) on day 3 of life. Oral milk feeds were usually started in the first 5 days of life at the discretion of the attending neonatologist and were given as intermittent boluses through an orogastric tube starting at $1 \mathrm{ml} /$ hour. Oral intake was increased cautiously at a rate of $0.5-1 \mathrm{ml} /$ hour/ day according to tolerance. Infants were fed mother's milk whenever possible, but preterm 
Table 1 Comparison of the clinical characteristics of the infants receiving oral erythromycin and those receiving placebo

\begin{tabular}{|c|c|c|}
\hline Clinical features & $\begin{array}{l}\text { Oral erythromycin group } \\
(n=27)\end{array}$ & $\begin{array}{l}\text { Placebo group } \\
(n=29)\end{array}$ \\
\hline Gestational age (weeks) & $29.6(28.6-30.7)$ & $29.3(27.5-31.0)$ \\
\hline Birth weight $(\mathrm{g})$ & $1180(985-1395)$ & $1160(1004-1389)$ \\
\hline Sex (female : male) & $12(44 \%): 15(56 \%)$ & $15(52 \%): 14(48 \%)$ \\
\hline Inborn : Outborn & $26(96 \%): 1(4 \%)$ & $28(97 \%): 1(3 \%)$ \\
\hline \multicolumn{3}{|l|}{ Mode of delivery ${ }^{\star}$} \\
\hline Vaginal & $6(22 \%)$ & $15(52 \%)$ \\
\hline Caesarean section & $20(74 \%)$ & $14(48 \%)$ \\
\hline Forceps & $1(4 \%)$ & $0(0 \%)$ \\
\hline \multicolumn{3}{|l|}{ Apgar scores } \\
\hline $1 \mathrm{~min}$ & $7(5-8)$ & $6(5-7)$ \\
\hline $5 \mathrm{~min}$ & $9(8-9)$ & $9(8-9)$ \\
\hline \multicolumn{3}{|l|}{ Umbilical arterial blood gas variables } \\
\hline $\mathrm{pH}$ & $7.29(7.23-7.32)$ & $7.29(7.19-7.32)$ \\
\hline Base excess & $-4.1(-5.6--2.3)$ & $-6.1(-9.0--0.8)$ \\
\hline First venous haematocrit after delivery & $0.52(0.44-0.54)$ & $0.50(0.42-0.54)$ \\
\hline Temperature on admission $\left({ }^{\circ} \mathrm{C}\right)$ & $36.2(36.0-36.6)$ & $36.3(36.0-36.8)$ \\
\hline \multicolumn{3}{|l|}{ Umbilical arterial catheter: } \\
\hline Infants with UAC (n) & $14(52 \%)$ & $18(62 \%)$ \\
\hline Duration (days) & $8(7-14)$ & $6.5(5-13)$ \\
\hline \multicolumn{3}{|l|}{ Umbilical venous catheter: } \\
\hline Infants with UVC (n) & $20(74 \%)$ & $23(79 \%)$ \\
\hline Duration (days) & $14(8-17)$ & $13.5(6-17)$ \\
\hline \multicolumn{3}{|l|}{ Respiratory distress syndrome } \\
\hline Grade $0-2$ & $14(52 \%)$ & $14(48 \%)$ \\
\hline Grade 3-4 & $13(48 \%)$ & $15(52 \%)$ \\
\hline \multicolumn{3}{|l|}{ Drugs } \\
\hline \multicolumn{3}{|l|}{ Antenatal dexamethasone } \\
\hline Number of mothers & $24(89 \%)$ & $25(86 \%)$ \\
\hline Doses $(n)$ & $2(1-4)$ & $2(1-4)$ \\
\hline \multicolumn{3}{|l|}{ Indomethacin } \\
\hline Number of infants & $10(37 \%)$ & $12(41 \%)$ \\
\hline \multicolumn{3}{|l|}{ Fentanyl } \\
\hline Number of infants & $22(82 \%)$ & $25(86 \%)$ \\
\hline Duration (days) & $5(1-7)$ & $3(1-11)$ \\
\hline \multicolumn{3}{|l|}{ Vecuronium } \\
\hline Number of infants & $8(30 \%)$ & $12(41 \%)$ \\
\hline Duration (days) & $0(0-2)$ & $0(0-2)$ \\
\hline
\end{tabular}

Continuous variables are expressed in median (interquartile ranges) and proportions in number of patients $(\%) ;{ }^{\star} \mathrm{p}<0.05$.

UAC, Umblical arterial catheter; UVC, umbilical venous catheter.

commercial milk formulas were also used if parents preferred. All infants were examined at least twice a day and closely monitored for the occurrence of emesis, diarrhoea, abdominal distension, and volume of gastric residuals. Strict guidelines were provided on stopping and restarting of enteral feeding. The attending neonatologist considered discontinuing enteral feeding when: vomiting occurred more than twice in 24 hours; the volume of gastric residuals exceeded half of the oral intake in the previous four hours on two occasions within the same day; the clinical signs and symptoms were

Table 2 Outcomes of enteral feeding and other clinical features of the two groups

\begin{tabular}{|c|c|c|}
\hline Outcomes & $\begin{array}{l}\text { Oral erythromycin } \\
\text { group }(n=27)\end{array}$ & $\begin{array}{l}\text { Placebo group } \\
(n=29)\end{array}$ \\
\hline Age started on enteral feeding (days) & $6(4-11)$ & $8(5-11)$ \\
\hline Volume of enteral feeding on enrollment (ml/kg/day) & $43(17-58)$ & $51(39-68)$ \\
\hline Time after enrollment achieved half enteral feeding (days) & $3.5(2-7)$ & $6(4-11.5)^{\star}$ \\
\hline \multicolumn{3}{|l|}{ Time after enrollment achieved three quarters enteral } \\
\hline Time after enrollment achieved full enteral feeding (days) & $13.5(8-22)$ & $25(16-33)^{\star \star}$ \\
\hline \multicolumn{3}{|l|}{ Type of milk feeds } \\
\hline Breast & $5(19 \%)$ & $3(10 \%)$ \\
\hline Formula & $8(30 \%)$ & $8(28 \%)$ \\
\hline Mixed & $14(51 \%)$ & $18(62 \%)$ \\
\hline Exchange tranfusion $(\mathrm{n})$ & $2(7 \%)$ & $2(7 \%)$ \\
\hline Patent ductus arteriosus (n) & $9(33 \%)$ & $13(45 \%)$ \\
\hline Necrotising enterocolitis (n) & $0(0 \%)$ & $0(0 \%)$ \\
\hline Duration of IPPV (days) & $5(3-11)$ & $5(2-15)$ \\
\hline Duration of mechanical ventilation (days) & $16(8-30)$ & $16(8-36)$ \\
\hline Duration of $\mathrm{O}_{2}$ dependence (days) & $18(8-34)$ & $16(8-36)$ \\
\hline \multicolumn{3}{|l|}{ Periventricular haemorrhage } \\
\hline Grade $0-2$ & $25(93 \%)$ & $26(90 \%)$ \\
\hline Grade 3-4 & $2(7 \%)$ & $3(10 \%)$ \\
\hline
\end{tabular}

Continuous variables are expressed in median (interquartile ranges) and proportions in number of patients $(\%) ;{ }^{\star} \mathrm{p}<0.05,{ }^{\star \star} \mathrm{p}<0.005$.

IPPV, Intermittent positive pressure ventilation. suggestive of necrotising enterocolitis or other ominous intra-abdominal pathologies; or repeated regurgitation and aspiration pneumonia were suspected. Oral feeding together with the study drug was resumed as soon as the above signs and symptoms subsided.

MICROBIOLOGICAL STUDIES

Stool samples for microbiological culture were collected from all infants studied immediately before treatment and immediately after and four weeks after the treatment had been stopped. All specimens were cultured on a wide range of selective media as previously described. ${ }^{17}$ Stool pathogens, including Salmonella spp, Shigella spp, thermophilic Campylobacter spp, and Vibrio spp, were identified using standard biochemical tests, the API systems (bioMérieux, Marcy-I' Etoile, France), and serological tests where appropriate. Heavy predominant growth and pure growth of aerobes, anaerobes, and fungi were recorded. The microbiologists who performed the stool culture were unaware of the randomisation assignments.

STATISTICAL ANALYSIS

Fisher's exact test, the Mann-Whitney U test, and the Wilcoxon rank sum test were used to compare proportions and continuous variables where appropriate. Spearman's correlation was also used to assess the relation between different variables that may affect enteral nutrition (table 1) and the time to achieve half, three quarters, and full enteral feeding after the drug treatment. All tests were performed by SPSS for Windows (Release 9.0; SPSS Inc, Chicago, Illinois, USA), and the level of significance was set at $5 \%$ for all comparisons. The results were analysed on an intention to treat basis.

ETHICS

The study was approved by the clinical research ethics committee of the Chinese University of Hong Kong, and informed parental consent was obtained before randomisation and enrolment.

\section{Results}

Ninety five VLBW infants were admitted to the neonatal intensive care unit during the 19 month period. Eight infants died from extreme prematurity and respiratory disease before 14 days of age, 21 infants received more than half of the total daily fluid volume by enteral feeding at 14 days of age and did not satisfy the entry criteria, and 10 parents did not give consent. Fifty six infants were enrolled in the study.

Twenty seven infants received oral erythromycin and 29 received the placebo solution. Tables 1 and 2 summarise the clinical characteristics and the time taken to reach different stages of enteral feeding after treatment respectively. The age at which enteral feeding was started and the milk intake ( $\mathrm{ml} / \mathrm{kg} /$ day) on the day of randomisation did not differ significantly between the two groups $(p=0.81$ and $p=0.11$ respectively). Infants in the oral erythromycin group achieved half, three quarters, and full enteral feeding significantly earlier 
Table 3 Comparison of the potential complications of prolonged total parenteral nutrition and erythromycin treatment between the oral erythromycin and the placebo group

\begin{tabular}{lll}
\hline Clinical features or complication & $\begin{array}{l}\text { Oral erythromycin } \\
\text { group }(n=27)\end{array}$ & $\begin{array}{l}\text { Placebo group } \\
(n=29)\end{array}$ \\
\hline QT interval before drug treatment (ms) & $0.37(0.34-0.38)$ & $0.37(0.34-0.40)$ \\
QT interval during drug treatment (ms) & $0.36(0.35-0.40)$ & $0.38(0.35-0.38)$ \\
Pyloric stenosis (n) & $0(0 \%)$ & $0(0 \%)$ \\
Septicaemia (no of episodes) & & 4 \\
$\quad$ Gram positive bacteria & 3 & 3 \\
$\quad$ Gram negative bacteria & 1 & 2 \\
$\quad$ Fungi & $10: 6: 2$ & $12: 9: 1$ \\
Stool culture (Gram positive : Gram negative : fungi) & $13: 6: 3$ & $11: 14: 1$ \\
$\quad$ Immediately before drug treatment & $5: 7: 0$ & $6: 11: 2$ \\
$\quad$ During drug treatment & $5(19 \%)$ & $10(35 \%)$ \\
$\quad 4$ weeks after drug treatment was stopped & $18(8-34)$ & $18(8-50)$ \\
Cholestatic jaundice (n) & $73(64-97)$ & $86(64-109)$ \\
Maximum serum conjugated bilirubin concentration (mmol/l) \\
Duration of hospital stay (days) & $0(0 \%)$ & $3(10 \%)$ \\
Number who died & & \\
\hline
\end{tabular}

Continuous variables are expressed in median (interquartile ranges) and proportions in numbers of patients $(\%)$.

than those in the placebo group $(\mathrm{p}<0.05$, $\mathrm{p}<0.05$ and $\mathrm{p}<0.0001$ respectively). By chance, more infants were born by caesarean section in the oral erythromycin group than in the placebo group $(\mathrm{p}<0.05)$. All other parameters did not differ significantly between the two groups (tables 1 and 2).

Table 3 summarises the potential adverse effects of erythromycin treatment and the potential complications of parenteral nutrition. The QT interval was not prolonged and did not differ significantly between the oral erythromycin and the placebo group before and during treatment. Although 10 of 29 infants in the placebo group and five of 27 infants in the oral erythromycin group developed cholestatic jaundice (serum conjugated bilirubin concentration $>34 \mathrm{mmol} / \mathrm{l}$ ) as the result of prolonged hyperalimentation, this trend did not reach significance $\left(\chi^{2}=1.82, p=0.18\right)$. None of the infants developed pyloric stenosis. All infants in the oral erythromycin group survived, but three infants in the placebo group died.

Eleven and nine episodes of septicaemia were documented in the oral erythromycin and the placebo group respectively. Coagulase negative staphylococcus (five cases), Staphylococcus aureus (one case), Enterococcus (one case), Klebsiella spp (one case), Escherichia coli (one case), Serratia spp (one case), and Candida parasilosis (one case) were isolated in the oral erythromycin group. Coagulase negative staphylococcus (four cases), Serratia spp (two cases), $E$ coli (one case), and $C$ albicans (two cases) were isolated in the placebo group. In addition, there was no significant difference in the stool culture pattern between the two groups of infants (table 3 ).

In the oral erythromycin group, there was significant association between the number of septicaemic episodes and the age at which half enteral feeding was established $(r=0.41$, $\mathrm{p}<0.05)$. There was also a significant association between the number of septicaemic episodes and the age at which three quarters enteral feeding was achieved $(r=0.48$, $\mathrm{p}<0.05)$ in the placebo group. There were, however, no significant correlations between the age at which half, three quarters, and full enteral feeding were reached and gestational age, birth weight, perinatal asphyxia indices, duration of umbilical arterial and venous catheterisation, patent ductus arteriosus, different drug treatment including antenatal corticosteroids, indomethacin, fentanyl, and paralysing agents, severity of respiratory distress syndrome, and periventricular haemorrhage.

\section{Discussion}

Our results indicate that VLBW infants with feed intolerance due to non-anatomically obstructive gastrointestinal dysmotility achieved full enteral feeding significantly earlier after treatment with oral erythromycin. The median times taken to establish half, three quarters, and full enteral nutrition were 3.5, 8.5 , and 13.5 days after the start of oral erythromycin. The median times taken to achieve half and full oral feeding in the placebo group were almost twice as long (table 2). These findings agree with those of previous case series in preterm infants. ${ }^{13-15}$ Although a recent randomised controlled study did not show any benefit of intravenous erythromycin in improving the tolerance of enteral feeding in preterm infants, ${ }^{25}$ these findings were not entirely unexpected because the study was not primarily designed to investigate the effectiveness of erythromycin on enteral feeding, but rather focused on the relation between chronic lung disease and intravenous erythromycin treatment for presumed Ureaplasma urealyticum infection. ${ }^{26}$ Even more important, the study did not specifically select infants with protracted feed intolerance who were in most need of prokinetic treatment. Therefore most of the patients did not have appreciable gastrointestinal dysmotility. In addition, an antimicrobial dose of intravenous erythromycin may not be ideal for producing its prokinetic effect and could even be dangerous as it may induce severe arrhythmia in preterm infants. ${ }^{18} 19$

Gestational age, birth weight, perinatal asphyxia indices, severity of the initial pulmonary disease, duration of umbilical arterial and venous catheterisation, and commonly used drugs such as antenatal corticosteroids, fentanyl, and paralysing agents were expected to influence tolerance to oral feeding; however, none were found to significantly affect the age at which full enteral nutrition was established. Septicaemia, however, did significantly delay enteral feeding as it was often associated with paralytic ileus and feed intolerance. Severely ill and septic infants may also inadvertently be kept nil by mouth for a prolonged period for fear of necrotising enterocolitis.

The potent prokinetic action of erythromycin has been shown to act principally at the level of the stomach and the proximal small bowel in both human and animal studies. ${ }^{327} 28$ There is substantial evidence to support the suggestion that erythromycin exerts its gastrointestinal motor effects through activation of the neural motilin receptors on cholinergic neurones and the smooth muscle motilin receptors of the upper gastrointestinal tract. ${ }^{29}$ Stimulation of the motilin pathway results in greater amplitude and more frequent antral contractions, ${ }^{70-32}$ an increase in proximal gastric tone, ${ }^{33}$ suppression of pyloric pressure 
waves, which is associated with reduced pyloric outlet resistance ${ }^{27}$ and an increase in duodenal contraction frequency. ${ }^{34}$ Human studies further suggest that low dose (1-3 $\mathrm{mg} / \mathrm{kg}$ ) erythromycin predominantly enhances the phase III migrating motor complex at the antral level, whereas high doses of the drug are mainly responsible for the production of sustained antral contractile activity and improved antroduodenal coordination. ${ }^{24} 2832$ In addition, a phase III-like motor pattern was observed with the higher dose of the drug. ${ }^{24}$ Combination of the aforementioned mechanisms is thus likely to produce powerful propulsive forces, which effectively propel the gastric luminal contents distally towards the small and large bowels and improve gastrointestinal motility. Despite some reports suggesting immaturity and a paucity of migrating contractile activity in infants below 32 weeks gestation, ${ }^{3536}$ our findings and the results of other studies indicate a dramatic improvement in milk tolerance and an increase in gastric emptying after introduction of oral or intravenous erythromycin in extremely premature and VLBW infants. ${ }^{13-16}$ In fact, the pattern of distribution of motilin in the gastrointestinal tract at 20 weeks gestation closely resembles adult patterns, and the development of the gastrointestinal neuroendocrine network is almost complete by 25 weeks gestation. ${ }^{35-37} \mathrm{It}$ has also been shown that infusion of exogenous motilin may promote an earlier appearance of the migrating motor complex, ${ }^{38}{ }^{39}$ and the introduction of enteral feeding to the neonatal gut has resulted in premature detection of phase III motor activity than would normally be expected for the gestational age. ${ }^{40}$ These studies illustrate the important concept that preterm infants are already equipped with the necessary anatomical and physiological apparatus at a very early gestation, and it is possible that erythromycin, a competitive analogue of motilin, can act on such motilin receptors and enhance upper gastrointestinal motility.

We admit that the design of the study is not perfect, as we were unable to find a good placebo substitute for erythromycin, which is a white liquid suspension with a distinct odour. However, we sought to overcome some of the difficulties of the blinding procedure by designating staff not involved in the daily care of the newborns to perform the randomisation procedure and to prepare the drugs and mix them into the milk feeds to mask their appearance. Therefore, the attending neonatologists, intensive care team, and microbiologists were completely unaware of the randomisation assignments and were truly blinded to the treatment given to these infants.

Our findings did not show a significant reduction in the incidence of cholestatic jaundice and systemic infection, despite the favourable outcome of achieving enteral feeding earlier in the oral erythromycin group. However, there was a trend suggesting that more infants with protracted feed intolerance developed cholestatic jaundice in the placebo group (table 3 ). As this study was principally designed to investigate the effectiveness of oral erythromycin in the promotion of enteral feeding, it may not have sufficient statistical power to detect small changes in associated benefits or complications. It is, however, reassuring that none of the infants in the oral erythromycin group developed cardiac dysrhythmia, pyloric stenosis, or septicaemia from multiresistant organisms. In addition, the patterns of the stool culture before, during, and after the treatment were very similar in the two groups. There were no deaths in the oral erythromycin group, but three infants died in the placebo group. One had severe chronic lung disease and died from respiratory failure. The other two had Gram negative septicaemia and died from multiorgan failure. Two of these infants never received more than three quarters of the total volume of enteral feed and none achieved full enteral feeding during the course of their illness.

In summary, this study shows that oral erythromycin is effective in facilitating enteral feeding in VLBW infants with moderately severe gastrointestinal dysmotility. Oral erythromycin can therefore be considered for use in preterm infants who fail to establish enteral feeding after an extended period and in whom an anatomically obstructive pathology of the gastrointestinal tract has been excluded. Although our results indicate that oral erythromycin at the dose used here is safe and does not appear to promote the emergence of resistant organisms, we caution against prophylactic or routine use of this treatment in preterm infants. Until the safety of erythromycin has been confirmed, this treatment should remain experimental, and liberal use of erythromycin as a prokinetic agent for mild cases of gastrointestinal dysmotility should be advised against. New macrolide analogues, such as ABT229, that are devoid of antibiotic activity but possess potent motilinomimetic properties are currently under intense investigation. ${ }^{41}{ }^{42}$ These new classes of drug may prove to be powerful prokinetics which are free from major adverse cardiac effects and will not promote the emergence of multiresistant organisms.

1 Pilot MA. Macrolides in roles beyond antibiotic therapy. $\mathrm{Br}$ 7 Surg 1994;81:1423-9.

2 Cucchiara S, Minella R, Scoppa A, et al. Antroduodenal motor effects of intravenous erythromycin in children with motor effects of intravenous erythromycin in children with abnormalities of gastrointestinal
Gastroenterol Nutr 1997;24:411-18.

3 Jadcherla SR, Klee G, Berseth CL. Regulation of migrating motor complexes by motilin and pancreatic polypeptide in human infants. Pediatr Res 1997;42:365-9.

4 Miller SM, O'Dorisio TM, Thomas FB, et al. Erythromycin exerts a prokinetic effect in patients with chronic idiopathic intestinal pseudoobstruction [abstract]. Gastroenterology 1990;98:A375.

5 Pennather A, Tran A, Cioppi M, et al. Erythromycin strengthens the hypotensive lower oesophageal sphincter in patients with gastroesophageal reflux disease. Am $\mathcal{F}$ Surg 1994;167:169-73.

6 Simkiss DE, Adams IP, Myrdal U, et al. Erythromycin in neonatal postoperative intestinal dysmotility. Arch Dis Child 1994;71:F128-9.

7 Jannssens J, Peeters T, Vantrappen G, et al. Improvement of gastric emptying in diabetic gastroparesis by erythromycin. N Engl fु Med 1990;322:1028-31.

8 Fiorucci S, Distrutti E, Gerli R, et al. Effect of acute and chronic administration of erythromycin on gastrointestinal motility in scleroderma patients [abstract]. Gastroenterology 1993;104:A507.

9 Hill AD, Walsh TN, Hamilton D, et al. Erythromycin improves emptying of the denervated stomach after improves emptying of the denervated ston

10 Di Lorenzo C, Lachman R, Hyman PE. Intravenous erythromycin for postpyloric intubation. $\mathcal{F}$ Pediatr Gastroenterol Nutr 1990;11:45-7. 
11 Colombo M, Magni LA. Jejunal biopsy with Watson's capsule and erythromycin. Minerva Pediatr 1995;47:97-8.

12 Stern MA, Wolf DC. Eryt prospective, randomized, controlled study of efficacy in nasoenteric tube placement. $A m \quad \mathcal{F}$ Gastroenterol 1994;89:2011-13.

$13 \mathrm{Ng}$ PC, Fok TF, Lee CH, et al. Erythromycin treatment for gastrointestinal dysmotility in preterm infants. $f$ Paediatr Child Health 1997;33:148-50.

$14 \mathrm{Su} \mathrm{BH}$, Lin HC, Peng CT, et al. Effect of erythromycin on feeding intolerance in very low birth weight infants: a preliminary observation. Acta Paediatr Sin 1998;39:324-6.

15 Kubota M, Nakamura T, Motokura T, et al. Erythromycin improves gastrointestinal motility in extremely low birthweight infants. Acta Paediatr fpn 1994;36:198-201.

16 Tomomasa T, Miyazaki M, Koizumi T, et al. Erythromycin increases gastric antral motility in human premature infants. Biol Neonate 1993;63:349-52.

17 Siu YK, Ng PC, Fung SCK, et al. Double blind, randomised, placebo controlled study of oral vancomycin in prevention of necrotising enterocolitis in preterm, very in prevention of necrotising enterocolitis in preterm, very low birth weight

18 Farrar HC, Walsh-Sukys MC, Kyllonen K, et al. Cardiac toxicity associated with intravenous erythromycin lactobionate: two case reports and a review of the literature. Pediatr Infect Dis $\mathcal{F}$ 1993;12:688-91.

19 Gouyon JB, Benoit A, Bétremieux P, et al. Cardiac toxicity of intravenous erythromycin lactobionate in preterm infants. Pediatr Infect Dis 7 1994;13:840-1.

20 Sande MA, Mandell GL. Antimicrobial agents. In: Gilman AG, Rall TW, Nies AS, et al, eds. Goodman and Gilman's the pharmacologic basis of therapeutics. 8th ed. Elmsford, NY: Pergamon, 1990:1130-4.

21 Parsons RL, David JA, Raymond K, et al. Pharmacokinetics on intravenous erythromycin lactobionate. F Int Med Res 1980;8(suppl 2):15-23.

22 Houin G, Tillement JP, Lhoste F, et al. Erythromycin pharmacokinetics in man. F Int Med Res 1980;8(suppl 2):9-14.

23 Gouyon JB. The use of erythromycin lactobionate to facilitate the postpyloric passage of tubes in children. $\mathcal{F}$ Pediat Gastroenterol Nutr 1992;14:120.

24 Annese V, Janssens J, Vantrappen G, et al. Erythromycin accelerates gastric emptying by inducing antral contractions and improved gastro-duodenal coordination. Gastroenterology 1992;102:823-8.

25 Stenson BJ, Middlemist L, Lyon AJ. Influence of erythromycin on establishment of feeding in preterm infants: observations from a randomised controlled trial. Arch Dis Child Fetal Neonatal Ed 1998;79:F212-14.

26 Lyon AJ, McColm J, Middlemist L, et al. Randomised tria of erythromycin on the development of chronic lun disease in preterm infants. Arch Dis Child Fetal Neonatal Ed 1998;78:F10-14.
27 Mathis C, Malbert $\mathrm{CH}$. Changes in pyloric resistance induced by erythromycin. Neurogastroenterol Motil $10: 131-8$

28 Coulie B, Tack J, Peeters T, et al. Involvement of two different pathways in the motor effects of erythromycin on the gastric antrum in humans. Gut 1998;43:395-400.

29 Parkman HP, Pagano AP, Vozzelli MA, et al. Gastrokinetic effects of erythromycin: myogenic and neurogenic mechanisms of action in rabbit stomach. Am $\mathcal{f}$ Physiol 1995;269:G418-26.

30 Holle GE, Steinbach E, Forth W. Effects of erythromycin in the dog upper gastrointestinal tract. Am $\mathcal{f}$ Physiol 1992;263: G52-9.

31 Otterson MF, Sarna SK. Gastrointestinal motor effects of erythromycin. Am F Physiol 1990;259:G355-63.

32 Tack J, Janssens J, Vantrappen G, et al. Effect of erythromycin on gastric motility in controls and in diabetic gastroparesis. Gastroenterology 1992;103:72-9.

33 Varannes SBD, Parys V, Ropert A, et al. Erythromycin enhances fasting and postprandial proximal gastric tone in humans. Gastroenterology 1995;109:32-9.

34 Fraser R, Shearer T, Fuller J, et al. Intravenous erythromycin overcomes small intestinal feedback on antral, pyloric, an duodenal motility Gastroenterology 1992;103:114-19.

35 Bisset WM, Watt JB, Rivers RPA, et al. Ontogeny of fasting small intestinal motor activity in the human infant. Gut 1988;29:483-8.

36 Berseth CL. Gestational evolution of small intestinal motility in preterm and term infants. F Pediatr 1989;115:64651 .

37 Amarnath RP, Berseth CL, Malagelada JR, et al. Postnatal maturation of small intestinal motility in preterm infants and term infants. Fournal of Gastrointestinal Motility 1989;1:138-43.

38 Vantrappen GR, Janssens J, Peeters TL, et al. Motilin and the interdigestive motor complex in man. Dig Dis Sci 1979;24:497-500

39 Janssens J, Hellemans J, Adrian TE, et al. Pancreatic polypeptide is not involved in the regulation of the migrating motor complex in man. Regul Pept 1982;3:41-9.

40 Berseth CL, Nordyke C. Enteral nutrients promote postnatal maturation of intestinal motor activity. Am $\mathcal{F}$ Physiol 1993;264:G1046-51.

41 Verhagen MAMT, Samsom M, Maes B, et al. Effects of a new motilide, ABT-229, on gastric emptying and postprandial antroduodenal motility in healthy volunteers. Aliment Pharmacol Ther 1997;11:1077-86.

42 Nieuwenhuijs VB, van Duijvenbode-Beumer H, Verheem A, et al. The effects of ABT-229 and octreotide on interdigestive small bowel motility, bacterial overgrowth and bacterial translocation in rats. Eur $\mathcal{f}$ Clin Invest 1999;29:33-40. 